\title{
Extraordinary field-aligned current signatures in Saturn's high-latitude magnetosphere: Analysis of Cassini data during Revolution 89
}

\author{
E. J. Bunce, ${ }^{1}$ S. W. H. Cowley, ${ }^{1}$ D. L. Talboys, ${ }^{1}$ M. K. Dougherty,${ }^{2}$ L. Lamy, ${ }^{2}$ \\ W. S. Kurth, ${ }^{3}$ P. Schippers, ${ }^{3}$ B. Cecconi, ${ }^{4}$ P. Zarka, ${ }^{4}$ C. S. Arridge,,${ }^{5,6}$ and A. J. Coates ${ }^{5,6}$ \\ Received 28 April 2010; revised 28 July 2010; accepted 4 August 2010; published 20 October 2010.
}

[1] During the periapsis pass of Revolution 89, specifically on day 291 of 2008 , the Cassini spacecraft observed unusual field-aligned current signatures in Saturn's high-latitude southern hemisphere in the midnight and dawn sector. The region of open field lines was found to be contracted close to the pole, and surrounded by an unusual region containing hot $\mathrm{keV}$ electrons and 'leading' field signatures indicative of super-corotating flow. Usual 'lagging' fields indicative of sub-corotating flow were also present at lower latitudes, though of unusual strength. Unique within the $\sim 40$ similar nightside auroral region Cassini passes during 2008, the overall field-aligned current system thus consisted of a central region of downward current flanked by two regions of upward current. This distinctive signature coincided with the first in situ encounter of Cassini with a source region of Saturn kilometric radiation, located within the unusual poleward region of upward current adjacent to the open-closed field line boundary. We propose that these unusual conditions relate to a major open flux closure event in Saturn's tail, possibly triggered by solar wind compression of the magnetosphere.

Citation: Bunce, E. J., et al. (2010), Extraordinary field-aligned current signatures in Saturn's high-latitude magnetosphere: Analysis of Cassini data during Revolution 89, J. Geophys. Res., 115, A10238, doi:10.1029/2010JA015612.

\section{Introduction}

[2] The high-latitude phases of the Cassini mission at Saturn have provided the first opportunity to study the fieldaligned currents that couple the magnetosphere, ionosphere, and upper atmosphere in a rotation-dominated gas giant planetary system [Bunce et al., 2008; Talboys et al., 2009a, 2009b]. Perturbations in the azimuthal component of the magnetic field indicate whether field lines are deflected out of meridian planes such that they 'lag' or 'lead' the direction of planetary rotation, while latitude gradients in the perturbation field indicate the direction and magnitude of the associated field-aligned currents. Lagging fields indicate angular momentum transfer from atmosphere to magnetosphere associated with plasma sub-corotation, while leading fields indicate reversed transfer associated with plasma super-

\footnotetext{
${ }^{1}$ Department of Physics \& Astronomy, University of Leicester, Leicester, UK.

${ }^{2}$ Blackett Laboratory, Imperial College, London, UK.

${ }^{3}$ Department of Physics and Astronomy, University of Iowa, Iowa City, Iowa, USA.

${ }^{4}$ LESIA, CNRS, Observatoire de Paris, Meudon, France.

${ }^{5}$ Mullard Space Science Laboratory, University College London, Dorking, UK

${ }^{6}$ Centre for Planetary Sciences at UCL/Birkbeck, London, UK.
}

corotation, the coupling taking place via ion-neutral collisions in the ionospheric Pedersen layer.

[3] Bunce et al. [2008] showed that in the southern summer near-noon sector the principal current is directed upward out of the ionosphere as the strongly lagging field in the open field region relaxes to smaller values on adjacent closed dayside field lines, and that this upward current maps to the ultraviolet (UV) auroral oval as viewed simultaneously by the Hubble Space Telescope (HST). Further study of the highlatitude Cassini data obtained in late 2006 and early 2007 by Talboys et al. [2009a] revealed that in a number of such dayside passes the sense of the field perturbations actually reverses to a leading configuration in a layer mapping to the outer dayside magnetosphere, such that the upward current region is flanked by downward currents at lower latitudes as the latter perturbation relaxes to smaller values further inside the magnetosphere. Somewhat different signatures were observed in the northern winter pre-dusk sector, with weak perturbations on open field lines giving way to lagging fields on outer closed field lines, associated with layers of downward then upward current flowing with increasing co-latitude from the pole. Similar behavior was found in a majority of cases in a survey of nightside field data by Talboys et al. [2009b], obtained by Cassini in 2008. However, additional interior layers of leading fields were again found in $\sim 25 \%$ of cases, such that the current pattern then consisted of a region of upward current flanked by two regions of downward current. 
[4] The 2008 high-latitude data set examined by Talboys et al. [2009b], which forms the background to the present study, corresponds to Cassini orbital revolutions (Revs) 5597 spanning January to December 2008. It contains a sequence of passes across both northern and southern nightside auroral regions at radial distances of $\sim 3-5 R_{S}$, with a near-equatorial periapsis occurring between. $\left(\mathrm{R}_{\mathrm{S}}\right.$ is Saturn's radius, equal to $60268 \mathrm{~km}$.) After Rev 97, the periapsis radius was significantly raised, such that subsequent data are no longer directly comparable. Allowing for some extended data gaps, the 2008 auroral region data set thus consists of 37 passes across the northern nightside auroral region and 39 across the southern, thus corresponding to 76 such passes in total. Examination shows that the field-aligned current signatures observed in the vast majority of these cases conform to the patterns described by Talboys et al. [2009b] as outlined above. However, one particular southern hemisphere pass stands out as exhibiting qualitatively different properties, which is thus of special significance in revealing the behavior of the system under unusual, one out of $\sim 40$, conditions. In this paper we therefore highlight this specific example, observed on day 291 of 2008 on Rev 89. In this case the usual lagging fields at auroral latitudes reversed to leading fields at very high latitudes in the presence of hot $\mathrm{keV}$ electrons, before lagging fields were again resumed on open field lines close to the pole. Uniquely within the 2008 data set, the current pattern in this case thus consisted of a region of downward current flanked by two regions of upward current. This distinctive signature also coincided with the first in situ encounter of the Cassini spacecraft with a source region of Saturn kilometric radiation (SKR) [Lamy et al., 2010], located within the unusual poleward layer of upward-directed current adjacent to the open-closed field line boundary.

\section{Cassini Trajectories}

[5] To exemplify the unusual nature of the signatures observed on Rev 89, we compare them with a more typical example observed on Rev 82, when Cassini traversed almost the same orbit near the noon-midnight meridian. The spacecraft trajectories are shown in Figure 1, with Rev 82 on the left and Rev 89 on the right. Figures $1 \mathrm{a}$ and $1 \mathrm{~b}$ show the periapsis portions of these orbits projected into the noon-midnight $\mathrm{X}-\mathrm{Z}$ plane, where $\mathrm{Z}$ is aligned with Saturn's rotation (and magnetic) axis and the $X-Z$ plane contains the Sun. The trajectory is shown by the solid line with dots at $3 \mathrm{~h}$ intervals, labeled with the day of year (DOY) number at the start of each day. Cassini passed over the nightside northern auroral region inbound and the southern auroral region outbound, crossing the equatorial plane between them at a local time (LT) of $\sim 23 \mathrm{~h}$ and a radial distance of $\sim 3 \mathrm{R}_{\mathrm{S}}$. In Figures $1 \mathrm{c}-1 \mathrm{f}$ we have projected the spacecraft position along model field lines into the northern and southern ionospheres for the intervals when Cassini was located north and south of the equator, respectively. The magnetic model employed consists of the "Cassini Saturn Orbit Insertion (SOI)' internal field model of Dougherty et al. [2005] and a typical ring current model derived by Bunce et al. [2007], as previously used for the same purpose by Bunce et al. [2008] and Talboys et al. [2009a, 2009b]. The mapped trajectories are projected onto a polar grid with dotted circles every $5^{\circ}$, viewed 'onto' the northern hemisphere in Figures 1c and 1d, and 'through' the planet in the southern hemisphere in Figures 1e and 1f. Midnight is on the left, and dawn at the bottom.

[6] The blue lines in Figures 1e and 1f show the median poleward and equatorward boundaries of the UV oval determined from a statistical study of southern hemisphere HST images by Badman et al. [2006]. These data are restricted mainly to dayside LTs due to limited nightside HST coverage. However, images obtained near southern summer solstice indicate nightside emissions centered near $\sim 20^{\circ}$ co-latitude [Grodent et al., 2005], indicated by the blue dashed lines. The corresponding northern hemisphere locations in Figures $1 \mathrm{c}$ and $1 \mathrm{~d}$ were obtained by mapping the southern boundaries along field lines into the northern hemisphere. They lie closer to the pole than in the south due to the quadrupole term in the internal field. It can be seen that on both Revs the spacecraft made near-perpendicular crossings through the auroral regions, in the pre-midnight sector on the northern hemisphere inbound passes, and in the midnight and post-midnight sector on the southern hemisphere outbound passes.

\section{Field, Plasma, and Radio Wave Signatures}

[7] In Figures $2 a$ and $2 b$ we present Cassini data from Revs 82 and 89 , respectively, each showing $36 \mathrm{~h}$ of data centered near periapsis. These show from top to bottom a radio spectrogram from the RPWS instrument [Gurnett et al., 2004], the integrated radio emission powers per unit solid angle over low (green) and high (blue) frequency bands covering the lower and upper bands of the SKR spectrum [see Lamy et al., 2008], an electron spectrogram $\sim 1 \mathrm{eV}-28 \mathrm{keV}$ from anode 5 of the CAPS-ELS spectrometer generally representing quasiisotropic magnetospheric electrons [Young et al., 2005], the three spherical polar components of the magnetic field referenced to the planet's northern spin and magnetic axis measured by the fluxgate magnetometer [Dougherty et al., 2004], and the spacecraft co-latitude mapped to the appropriate ionosphere, $\theta_{i}$, as in Figure 1. The data at the bottom of Figures $2 \mathrm{a}-2 \mathrm{~b}$ show the universal time (hours), the spacecraft position, specifically the LT (decimal hours), the co-latitude with respect to the northern spin axis (deg), and the radial distance $\left(\mathrm{R}_{\mathrm{S}}\right)$. We note that in the panel showing the integrated radio powers, the high frequency band (blue) is 40 $1000 \mathrm{kHz}$ in both cases, while the low frequency band (green) is $20-40 \mathrm{kHz}$ for Rev 82 and $10-40 \mathrm{kHz}$ for Rev 89 . The lowest frequencies are omitted for Rev 82 in order to exclude contributions of low frequency non-SKR emissions. In the magnetic field panels the radial $(r)$ and co-latitudinal $(\theta)$ field components shown are residuals having the 'Cassini SOI' internal field model subtracted, while the azimuthal component $(\varphi)$ is as measured, since the model planetary field is axi-symmetric with no azimuthal component. Physical region identifiers based on the large-scale field-aligned current signatures observed in the azimuthal field component are also shown in the latter panel, delimited by the vertical dotted lines, labeled $a-h$ in Figure 2a and $a-l$ in Figure 2b. We also note that in the ELS spectrogram the interval of high electron counts across all energies observed in the vicinity of the equatorial crossing, marked by the vertical dashed line, is an artifact due to penetrating radiation belt particles, while the electrons observed below a few tens of $\mathrm{eV}$ at co-latitudes less than $\sim 18^{\circ}$ with respect to either pole where the spacecraft 

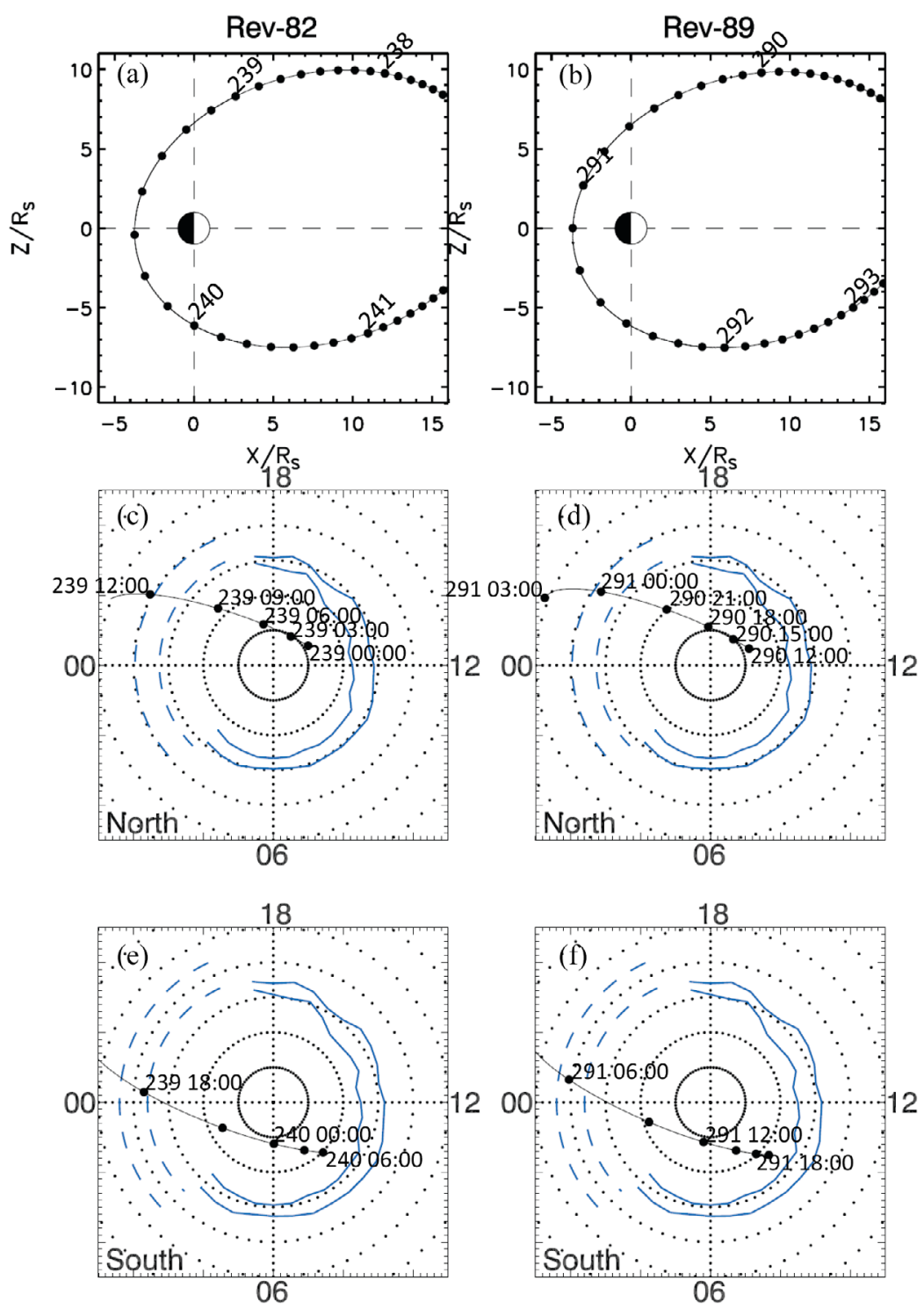

Figure 1. Trajectory of Cassini (black lines) on Revs (left) 82 and (right) 89 showing projections onto ( $a$ and $b$ ) the noon-midnight $X-Z$ plane, (c and d) along field lines into the northern ionosphere, and (e and f) along field lines into the southern ionosphere, viewed through the planet. The blue lines in Figures 1c-1f indicate auroral boundaries, specifically the solid lines show the median dayside UV oval determined from HST images by Badman et al. [2006], while the dashed lines show the nightside emissions indicated by Grodent et al. [2005].

potential is positive (e.g., the open field region), are electrostatically trapped spacecraft photoelectrons.

\subsection{Rev 82 Data}

[8] We first examine the field and particle data for Rev 82 shown in Figure 2a. The residual $r$ and $\theta$ components of the magnetic field principally show the effect of Saturn's ring current, with small auroral zone fluctuations superposed. The azimuthal component, however, principally shows the presence of antisymmetric lagging fields peaking at $\sim 10-15 \mathrm{nT}$ at auroral latitudes in both hemispheres, negative in the north (regions $b$ and $c$ ) and positive in the south (regions $f$ and $g$ ). Weaker slowly varying azimuthal fields are observed elsewhere, both on open field lines at highest latitudes (regions $a$ and $h$ identified via the lack of warm or hot electrons, corresponding to the blue regions in the electron spectrogram above a few tens of $\mathrm{eV}$ ), and at lower latitudes spanning the equator (regions $d$ and $e$ ). As indicated in section 1, this azimuthal field pattern is typical of the majority of the 2008 data reported by Talboys et al. [2009b]. The directions of the main field-aligned currents are indicated by the arrows, up or down with respect to the corresponding ionosphere. With increasing distance from the poles the currents are directed downward and then upward in both hemispheres. The downward-directed currents (regions $b$ and $g$ ) are co-located with weak variable fluxes of $\sim$ few $100 \mathrm{eV}$ electrons (green and yellow in the spectrogram) taken to correspond to the outer magnetosphere located just equatorward of the openclosed field line boundary, while the upward currents (regions $c$ and $f$ ) correspond to the usual outer ring current regime 
(a) Rev-82

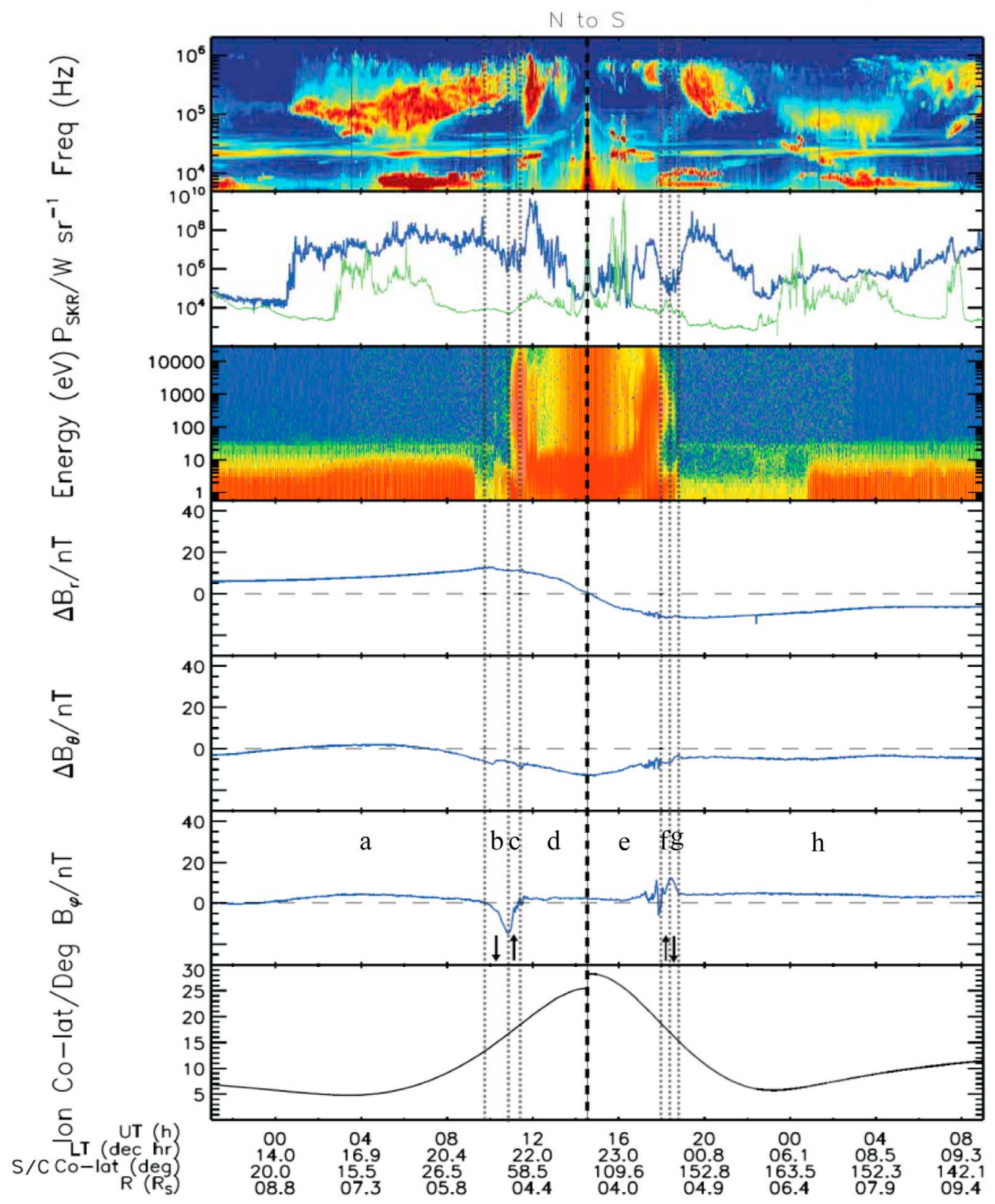

Figure 2. Plots of 36 h of Cassini data for (a) Rev 82 and (b) Rev 89, showing from top to bottom a radio spectrogram, integrated radio powers per unit solid angle over $40-1000 \mathrm{kHz}$ (blue) and $20-40 \mathrm{kHz}$ (for Rev 82) or 10-40 kHz (for Rev 89) (green), an electron spectrogram, three spherical polar components of the magnetic field referenced to the north polar axis of the planet with internal field subtracted, and the spacecraft co-latitude mapped to the corresponding ionosphere. The data at the bottom of each figure show the universal time (hours), the spacecraft position, specifically the LT (decimal hours), the co-latitude with respect to the northern spin axis (deg), and the radial distance $\left(\mathrm{R}_{\mathrm{S}}\right)$. The intervals shown are from $21 \mathrm{UT}$ on day 238 (of 2008) to 09 UT on day 240 for Rev 82, and from 09 UT on day 290 to 21 UT on day 291. Region identifiers $a-h$ (Figure 2a) and $a-l$ (Figure 2b) are shown in the azimuthal field $(\varphi)$ panel, delimited by the vertical dotted lines.

characterized by more intense electron fluxes (yellow and red) spanning a broad range of energies extending toward the top of the ELS energy band [e.g., see Schippers et al., 2008].

[9] The observed perturbations in the azimuthal magnetic field can be combined with Ampère's law to determine the total field-aligned current flowing in each current layer. If we consider the azimuthal field $B_{\varphi}$ observed at some point between the ionosphere and the closure currents in the nearequatorial magnetosphere, then assuming approximate axisymmetry, application of Ampère's law to a circular loop 
(b) Rev-89

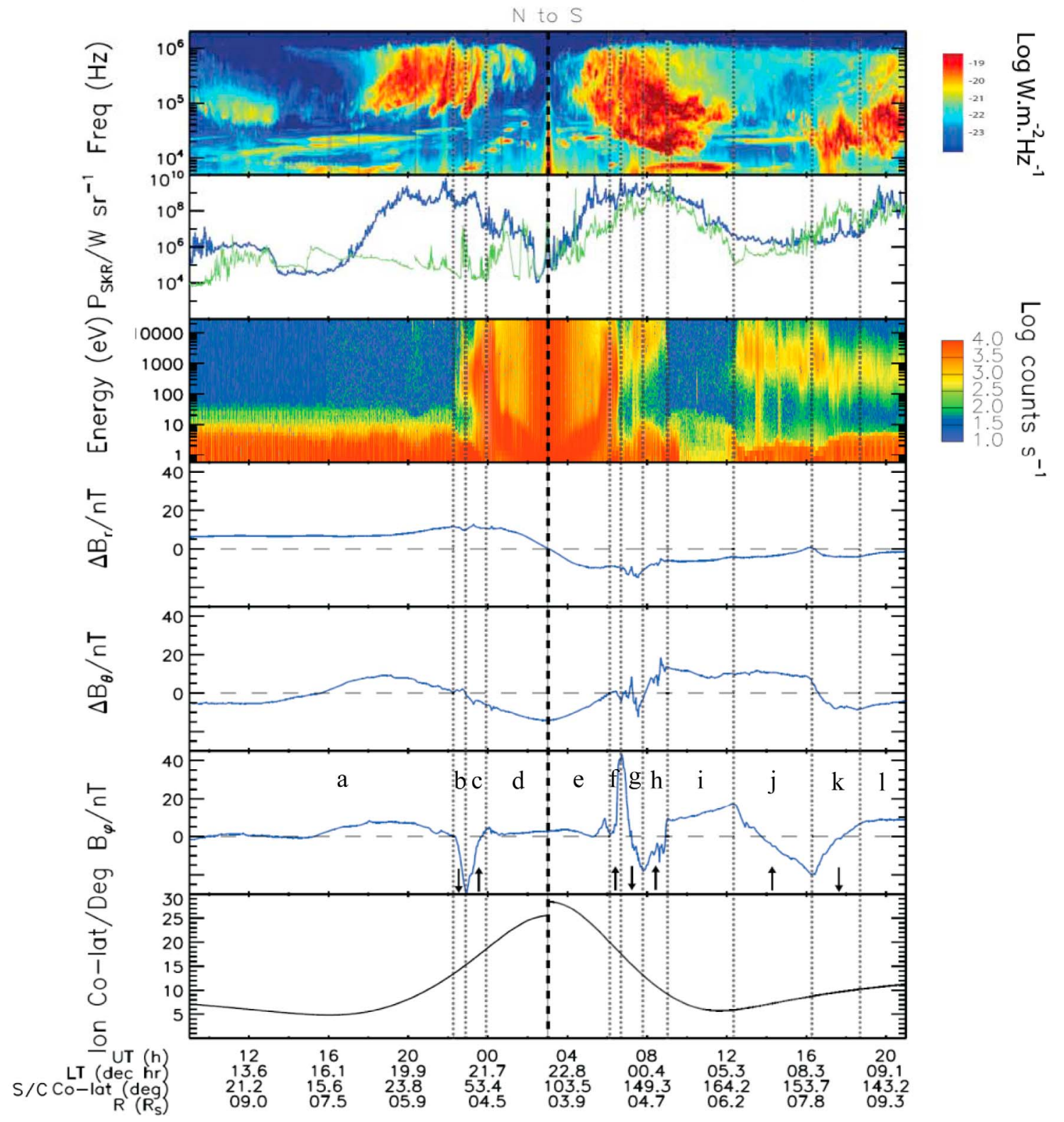

Figure 2. (continued)

passing through the point and centered on the magnetic axis shows that the azimuthal field is given by

$$
B_{\varphi}=\mp\left(\mu_{0} I_{P} / \rho\right) .
$$

In this expression $I_{P}$ is the ionospheric Pedersen current per radian of azimuth flowing at the feet of the field lines considered, taken to be positive when directed equatorward in both hemispheres, $\rho$ is the perpendicular distance from the axis of symmetry, and the upper sign is appropriate to the northern hemisphere and the lower to the southern hemisphere (see Talboys et al. [2009a] for further details). Using this equation we can then use the observations of $B_{\varphi}$ combined with the axial distance of the spacecraft $\rho$ to estimate $I_{P}$, and hence the field-aligned currents flowing. If, for example, the equatorward Pedersen current increases with increasing co-latitude from $I_{P}$ to $I_{P}+\Delta I_{P}$, then from current continuity a field-aligned current $\Delta I_{P}$ must flow down the field lines between the two locations. Using equation (1) with the data shown in Figure 2a, the total downward and upward currents are 2.5 MA per radian of azimuth in the north (regions $\mathrm{b}$ and $\mathrm{c}$ ), centered at ionospheric co-latitudes $\theta_{i}$ $\sim 15^{\circ}$ and $\sim 17.5^{\circ}$, respectively, and $\sim 1 \mathrm{MA} \mathrm{rad}^{-1}$ in the south (regions $\mathrm{f}$ and $\mathrm{g}$ ), centered at $\theta_{i} \sim 15.5^{\circ}$ and $\sim 17.5^{\circ}$. 
[10] Turning now to the radio data in the upper two panels of Figure $2 \mathrm{a}$, we note that the SKR emissions exhibit no unusual properties during this pass. The spectrum extends down in frequency only to several tens of $\mathrm{kHz}$, with typical $40-1000 \mathrm{kHz}$ integrated powers per unit solid angle (blue line in the second panel) of a few times $10^{7} \mathrm{~W} \mathrm{sr}^{-1}$, comparable with the lower $\sim 50 \%$ quantile value of integrated SKR powers of $\sim 2 \times 10^{7} \mathrm{~W} \mathrm{sr}^{-1}$ determined by Lamy et al. [2008]. The power in the low frequency $20-40 \mathrm{kHz}$ band (green line in the second panel) remains small, except for the sporadic appearance of non-SKR emissions. Previous work has shown that enhancements in the integrated SKR power per unit solid angle to $\sim 10^{9} \mathrm{~W} \mathrm{sr}^{-1}$, with extension of the spectrum below $\sim 10 \mathrm{kHz}$, are indicative of solar wind compressions of the magnetosphere and tail dynamics [Kurth et al., 2005; Jackman et al., 2005, 2009; Badman et al., 2008; Clarke et al., 2009]. The absence of such enhancements on Rev 82 indicates that the magnetosphere was 'quiet' on this pass. Unfortunately, for the interval covering both Revs 82 and 89 it is not appropriate to employ solar wind parameters propagated from near the Earth to the orbit of Saturn due to the azimuthal separation of Earth and Saturn at this time (K. C. Hansen, personal communication, 2010). As a result, we can only use the SKR power and spectrum as approximate proxies for possible solar wind-induced active conditions during these passes, based on the above-cited studies.

\subsection{Rev 89 Data}

[11] We now turn to the field and particle data for Rev 89 in Figure 2b. Conditions on the northern inbound pass were similar to Rev 82, with lagging azimuthal fields on outer closed field lines adjacent to the open field boundary (regions $b$ and $c$ ). However, these fields were significantly stronger than on Rev 82 , peaking at $\sim 30 \mathrm{nT}$ at $\theta_{i} \sim 15.5^{\circ}$, such that the downward and upward currents were increased to $~ 5 \mathrm{MA}$ $\operatorname{rad}^{-1}$ centered at $\theta_{i} \sim 14.5^{\circ}$ and $17^{\circ}$, respectively. The usual lagging field region was also observed on the southern outbound pass in regions $f$ and the first part of $g$, though again of unusual strength, with peak positive perturbations of $\sim 40 \mathrm{nT}$ at $\theta_{i} \sim 17.5^{\circ}$, the largest such field observed during the $\sim 40$ passes of the 2008 data set. However, as the spacecraft moved to higher southern latitudes, this perturbation did not relax to weak positive lagging fields on open field lines as on Rev 82. Instead, it reversed to form a region of negative leading fields (shown in the latter part of $g$ and in region $h$ ) peaking at a magnitude of $\sim 20 \mathrm{nT}$ at $\theta_{i} \sim 13^{\circ}$, in the presence of an unusual high-latitude hot electron population extending to the highest energies measured by the ELS instrument. We also note the presence of perturbations in the $\theta$ field component superposed on the ring current field, showing that the perturbations were not entirely azimuthal in this case. The spacecraft then crossed onto open field lines at $\theta_{i} \sim 9^{\circ}$ and $\sim 1 \mathrm{~h} \mathrm{LT}$ (region $i$ ), where the azimuthal field reversed to positive lagging values of $\sim 10 \mathrm{nT}$. Unique within the 2008 data set, the current system thus consisted of a central region of downward current flanked by two regions of upward current. The usual equatorward upward current region carried a total current of $\sim 5 \mathrm{MA} \mathrm{rad}^{-1}$ centered at $\theta_{i} \sim 18.0^{\circ}(\operatorname{region} f)$. The central downward current then carried $\sim 8 \mathrm{MA} \mathrm{rad}^{-1}$ centered at $\theta_{i} \sim 15.0^{\circ}$ (region $g$ ), while the unusual poleward upward current adjacent to the open field region carried $\sim 3 \mathrm{MA} \mathrm{rad}^{-1}$ centered at $\theta_{i} \sim 11.0^{\circ}$ (region $h$ ).
[12] Cassini then remained on open field lines for $\sim 3 \mathrm{~h}$ as it moved toward and away from its smallest southern colatitude of $\theta_{i} \sim 5.5^{\circ}$ (see Figure 1f), then re-encountering closed field lines and hot (now few-keV) electrons at $\theta_{i} \sim 6^{\circ}$ and $\sim 5 \mathrm{~h} \mathrm{LT}$ at the start of region $i$. Throughout this interval, therefore, the open field region in the post-midnight sector was contracted considerably poleward of its usual location of $\theta_{i} \sim 16^{\circ}$ (e.g., Figure 2a). On re-entering the closed field region, the azimuthal component again declined to negative leading fields of $\sim 20 \mathrm{nT}$ peak magnitude, implying the presence of total upward currents of $\sim 5 \mathrm{MA} \mathrm{rad}^{-1}$ centered at $\theta_{i} \sim 7.5^{\circ}$. Toward the end of the interval in Figure $2 \mathrm{~b}$ the azimuthal field then increased to positive lagging values on closed field lines as Cassini moved to larger co-latitudes in the pre-noon sector (region $k$ ), implying the presence of a similar downward current of $\sim 5 \mathrm{MA} \mathrm{rad}^{-1}$ centered at $\theta_{i}$ $\sim 9.5^{\circ}$. As the azimuthal field switched to positive values the electron energy declined to a few hundred $\mathrm{eV}$ (region $l$ ), more typical of Saturn's outer closed-field magnetosphere.

[13] Now examining the radio data on this pass, we note that the higher frequency integrated SKR powers were enhanced to $\sim 10^{9} \mathrm{~W} \mathrm{sr}^{-1}$ both inbound and outbound, while on the outbound pass the spectrum also extended down to much lower frequencies of $\sim 10 \mathrm{kHz}$, with large powers up to $\sim 10^{9} \mathrm{~W} \mathrm{sr}^{-1}$ also occurring in the low frequency $10-40 \mathrm{kHz}$ band. We note that it was in the latter high-power region (interval $h$ ) near $\sim 09$ UT on day 291 that the low-frequency limit of the SKR emission extended just below the local electron gyrofrequency, showing that the spacecraft encountered an SKR source region in situ [Lamy et al., 2010]. The emission flux density at $10 \mathrm{kHz}$ at that time reached $10^{-18} \mathrm{~W}$ $\mathrm{m}^{-2} \mathrm{~Hz}^{-1}$, within the upper $1 \%$ quantile for SKR observations at higher latitudes [Lamy et al., 2008]. The results presented here thus show that this SKR source was embedded within the unusual high-latitude region of upward-directed fieldaligned currents and hot keV electrons bordering the contracted region of open field lines. Observations of lower frequency Z-mode waves and auroral hiss in this interval have also been presented by Ye et al. [2010] and Kopf et al. [2010], respectively.

[14] Overall, the elevated SKR power and low-frequency extension of the spectrum, combined with the poleward contraction of the open-closed field line boundary in the dawn sector, together strongly suggest that these data represent the in situ observation of a dynamic dawn sector auroral contraction event similar to those observed remotely during HST auroral imaging campaigns [Clarke et al., 2005, 2009; Kurth et al., 2005], which are known to correspond to solar wind compressions of the magnetosphere. Jackman et al. [2005, 2009] and Badman et al. [2008] have also shown more generally that SKR power enhancements and low frequency extensions such as those observed here are associated with solar wind compressions and tail dynamics. On this basis we thus suggest that the unusual currents and electron populations observed on this pass may also be so related. It is nevertheless difficult using this data set alone to determine exactly when such a compression may have occurred, due e.g., to the lack of visibility of the SKR sources during the preceding periapsis interval. However, the proposed compression must have occurred prior to $\sim 06$ UT on day 291, after which high-power low-frequency SKR emissions became continuous for several hours. 

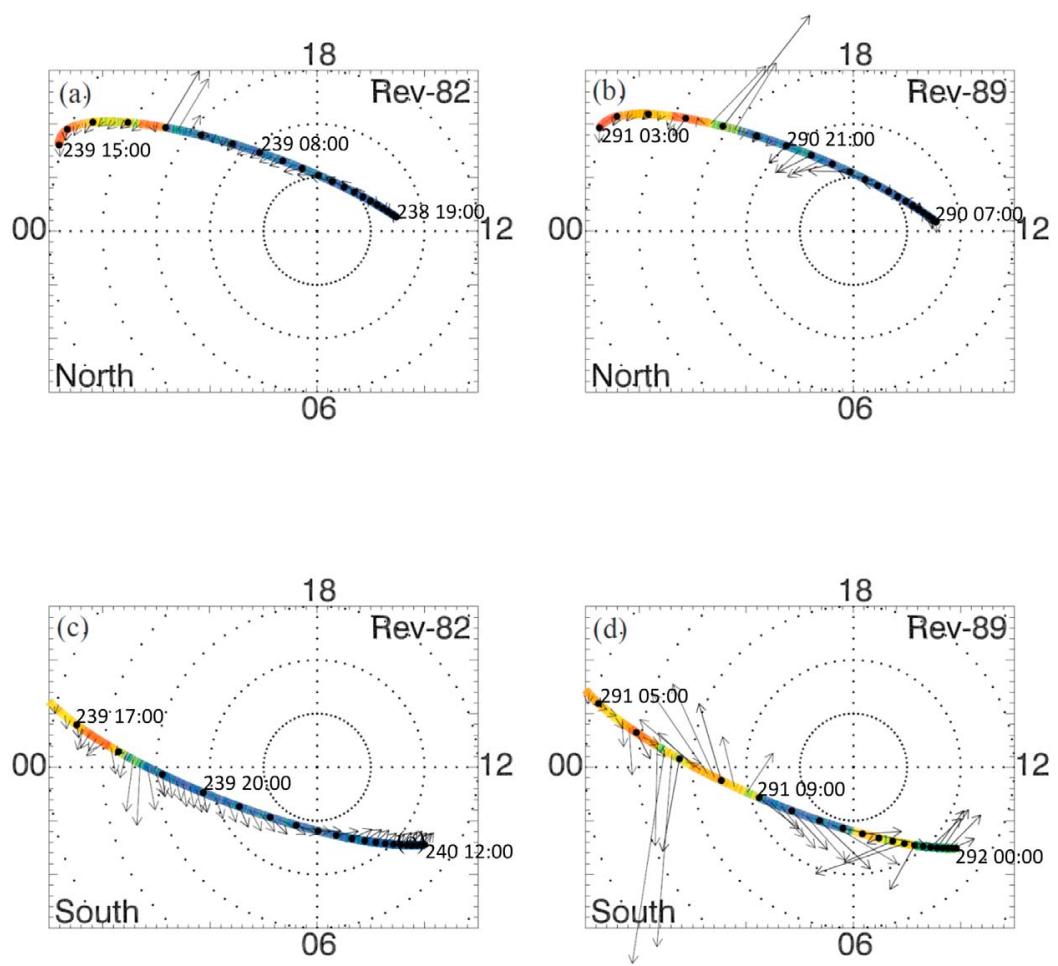

Figure 3. Perturbation magnetic field vectors (black arrows) are shown plotted along the spacecraft track projected into the ( $a$ and b) north and ( $c$ and d) south ionosphere (as in Figure 1) for Rev (left) 82 and (right) 89. Lines of constant latitude are shown at $5^{\circ}$ intervals. The spacecraft track is color-coded with the $5 \mathrm{keV}$ electron flux using the same scale as in Figure 2, with black dots being shown at $1 \mathrm{~h}$ time intervals. The field vectors shown are instantaneous $1 \mathrm{~min}$ vectors, plotted at approximately equal $\sim 1000 \mathrm{~km}$ increments along the projected spacecraft track.

\subsection{Perturbation Field Vectors}

[15] In Figure 3 we represent the perturbation fields observed on Revs 82 and 89 as vectors plotted along the spacecraft track projected into the appropriate ionosphere, in a format similar to the lower panels of Figure 1. The vectors have been obtained from the $\theta$ and $\varphi$ components by first removing the slow background field variations by low-pass filtering the data with a cut-off at $6 \mathrm{~h}$ (much longer than the variations of interest), and subtracting this field from the data. Instantaneous $1 \mathrm{~min}$ vectors are then plotted at approximately equal $\sim 1000 \mathrm{~km}$ increments of distance along the projected track, a vector corresponding to $10 \mathrm{nT}$ having a length equivalent to the radial distance between the $5^{\circ}$ circles. To give an indication of the plasma regime, we have color-coded the spacecraft track with the count rate of $\sim 5 \mathrm{keV}$ electrons using the same scale as in Figure 2. Dark blue colors thus correspond to the open field region, while greens through reds correspond to higher-flux regions on closed field lines.

[16] To a first approximation the perturbation vectors indicate the direction of ionospheric plasma flow relative to the neutral atmosphere. The field perturbations are produced by drag due to ion-neutral collisions in the Pedersenconducting layer, which causes the vectors above the ionosphere to tilt in the direction of the relative motion in the north, and opposite to the direction of relative motion in the south. Thus sub-corotating plasma flow gives rise to lagging vectors directed clockwise (negative azimuthal component) in the north and anti-clockwise (positive azimuthal component) in the south, as viewed in Figure 3. For Rev 82 (Figures $3 \mathrm{a}$ and $3 \mathrm{c}$ ) such fields are evident just equatorward of the open field boundary in both hemispheres, and at lower magnitudes in the southern open field region. Similar perturbations, but of larger magnitude, are observed on the northern inbound pass of Rev 89 (Figure 3b). Larger lagging fields may relate either to slower plasma flow relative to rigid corotation or to larger ionospheric Pedersen conductivity, both of which will increase the drag force and hence the tilt of the field. Figure $3 \mathrm{~d}$ then illustrates the very different conditions observed during the southern outbound pass of Rev 89. The open region with lagging fields indicating sub-corotating flow is contracted toward the pole in the nightside and dawn sector, surrounded by a layer of hot electrons and leading fields indicating super-corotating flow, with indications of equatorward directed field vectors and hence poleward flow near to midnight. These conditions give way to usual but large-amplitude lagging fields indicating sub-corotating flow at lower latitudes.

\section{Discussion and Summary}

[17] The results in section 3 demonstrate the unusual features of the Cassini observations during the southern outbound pass of Rev 89, unique within the ensemble of 37 northern and 39 southern similar passes of the spacecraft across Saturn's nightside auroral region during 2008. In 
essence, these features consist of a layer of hot electrons and 'leading' fields which extends poleward of the usual ring current population to a strongly contracted open field boundary, the latter regions containing usual 'lagging' fields. The overall large-scale field-aligned current system thus uniquely consists of a central downward current flanked by two upward-directed currents in this case. Concurrent radio observations show an enhancement of the emitted SKR power and an extension of the spectrum to low frequencies. Previous work has shown that such radio observations are related to solar wind compressions of the magnetosphere and tail dynamics [Kurth et al., 2005; Jackman et al., 2005, 2009; Badman et al., 2008], which have also been shown to be associated with bright auroral forms extending poleward of the usual oval in the dawn sector [Clarke et al., 2005, 2009], to which we relate the present in situ observations. Indeed, Clarke et al. [2005] have shown that these dawn auroras evolve into spiral structures around the pole, while Lamy et al. [2010] have shown that the SKR sources on this pass also form an unusual high-latitude spiral in the southern dawn sector. These features seem clearly related to the unusual high-latitude field-aligned current and plasma conditions reported here.

[18] Cowley et al. [2005] suggested that such events result from compression-induced bursts of reconnection and flux closure in Saturn's magnetic tail, similar to events that are observed occasionally at Earth [e.g., Boudouridis et al., 2004]. Following reconnection in the tail, the resulting newly closed flux tubes and hot plasma, overlying the previously existing trapped populations, flow toward the planet under the action of magnetic tension forces, and rotate with the planet via dawn. We propose that this scenario applies to the Rev 89 event described here. The amount of open flux closed during the event is readily estimated from the displacement of the open-closed boundary from its usual location. Using the same magnetic model as employed in section 2 above, a contraction of the southern boundary from $\sim 16^{\circ}$ (consistent with Figure $2 \mathrm{a}$ and Talboys et al. [2009b]) to $\sim 6^{\circ}$ as indicated by our results, implies the closure of $\sim 15 \mathrm{GWb}$ of magnetic flux. This assumes that the poleward contraction is confined to the dawn hemisphere as indicated e.g., by the results of Clarke et al. [2005, 2009].

[19] Given the association suggested here of this event with the occurrence of high-latitude dawn auroras such as those observed by Clarke et al. [2005, 2009], it is of interest to consider the high-latitude hot electrons observed on Rev 89 in relation to auroral emissions. Integration over the observed distributions, however, indicates precipitating energy fluxes of only $\sim 0.1 \mathrm{~mW} \mathrm{~m}^{-2}$, capable of producing UV emissions of $\sim 1 \mathrm{kR}$, comparable to the HST threshold. Precipitation of the observed hot electrons will not, therefore, produce bright UV auroras (typically 10s of kR) directly. However, these electrons may be accelerated along the field lines beneath the spacecraft to produce greater energy fluxes at the ionosphere if so required by the upward-directed field-aligned currents flowing. Here we thus consider the three regions of upward current observed on the outbound pass, namely that associated with the lower latitude region of lagging field (region $f$ in Figure 2b), and those associated with the high-latitude region of leading field that was traversed twice (regions $h$ and $j$ ), in the post-midnight and post-dawn sectors. Mapping the upward currents to the ionosphere along the field yields current densities in these regions of $\sim 280, \sim 65$, and $\sim 300 \mathrm{nA}$ $\mathrm{m}^{-2}$, all of which are significantly larger than the estimated local current densities of the downgoing portion of the hot $\mathrm{keV}$ electrons in these regions, typically a few tens of $\mathrm{nA} \mathrm{m}^{-2}$. Field-aligned acceleration of these electrons is therefore required to carry the observed currents. Application of Knight's [1973] kinetic theory to these values then suggests acceleration voltages of a few tens of $\mathrm{kV}$ in each case, yielding precipitating electron energy fluxes of $\sim 5, \sim 3$, and $\sim 20 \mathrm{~mW} \mathrm{~m}^{-2}$, which will produce bright UV auroras of $\sim 50$, $\sim 30$, and $\sim 200 \mathrm{kR}$ (see Bunce et al. [2008] for further details of such estimates). Although no UV observations are available during this event, bright dawn auroras are thus nevertheless anticipated, both in the main oval (the upward current layer in lagging field region $f$ at lower latitudes), and at high latitudes adjacent to the contracted region of open field lines (regions $h$ and $j$ ).

[20] We finally note that the SKR source region that was encountered in situ by Cassini on this pass, as reported by Lamy et al. [2010], lay within the unusual poleward region of upward-directed field-aligned current (region $h$ ) containing hot electrons where the background plasma frequency was much less than the gyrofrequency (close to the local frequency of SKR emission). However, it has been shown by Lamy et al. [2009] that under more usual conditions the SKR sources correspond to the usual lower-latitude UV auroral oval, which we suppose corresponds to the usual lowerlatitude region of upward-directed field-aligned current (regions $c$ and $f$ on both Revs in Figure 2) that typically lies within the outer ring current region. No in situ observations of the usual SKR source regions have yet been obtained, which may have distinctly different properties from those observed under the unusual physical conditions on Rev 89. In situ observations of these SKR sources presumably requires measurements on auroral flux tubes at considerably lower radial distances than those reported here $\left(\sim 5 \mathrm{R}_{\mathrm{S}}\right)$, below $\sim 2.5 \mathrm{R}_{\mathrm{S}}$ judging from the usual low-frequency cut-off observed.

[21] In summary, our principal findings are as follows.

[22] 1 . On the southern outbound pass of Cassini Rev 89 we observed a sequence of high-latitude field-aligned current signatures that are both of unusual strength and qualitatively different in form to those observed on all similar auroral region passes during 2008, the comparative data set consisting of 37 passes across the northern auroral region in the premidnight sector and 39 passes across the southern auroral region in the midnight and post-midnight sector.

[23] 2. During this pass, the boundary of open field lines was strongly contracted toward the pole, to $\sim 6^{\circ}$ co-latitude compared with $\sim 16^{\circ}$, indicative of a major episode of open flux closure in Saturn's tail lobes, estimated to be $\sim 15 \mathrm{GWb}$.

[24] 3. The contracted open field region with lagging (positive) azimuthal fields was surrounded by an unusual high-latitude region containing hot $\mathrm{keV}$ electrons with leading (negative) azimuthal fields, which extended equatorward to the usual region of lagging fields mapping to the outer ring current.

[25] 4. The field-aligned current pattern, unique within the 2008 Cassini data set, thus consisted of a central layer of downward-directed current flanked by two regions of upward-directed current. The lower-latitude upward current corresponded to the usual region of lagging field but here considerably enhanced in strength, while the unique high- 
latitude upward current was associated with leading fields adjacent to the open field region.

[26] 5. The pass occurred during an interval of enhanced SKR emissions extending to low frequencies whose sources form an unusual high-latitude spiral in the dawn sector [Lamy et al., 2010] that appears to correspond to the unusual current and plasma regimes reported here. The encounter with the SKR source reported by these authors occurred within the unusual high-latitude region of upward-directed current and hot electrons.

[27] 6. While the hot keV electron population observed at high-latitudes has insufficient energy flux to produce bright UV auroras directly, such emissions are anticipated in regions of upward-directed field-aligned current following downward field-aligned acceleration of these electrons.

[28] 7. Comparison of these properties with previously published results, specifically the poleward expansion of dawn sector closed field lines combined with the SKR enhancement and low-frequency extension, suggests that these observations relate to a major episode of open flux closure in Saturn's tail, that may have been triggered by a solar wind compression of the magnetosphere.

[29] Acknowledgments. EJB was supported by STFC grant PP/ E001130/1, DLT by STFC grant SG PP/D002117/1, CSA by STFC Postdoctoral Fellowship ST/G007462/1, CSA and AJC by the STFC rolling grant to MSSL/UCL, SWHC by STFC grants PPA/E000983/1 and ST/H002480/1, and MKD by STFC funding to Imperial College. We thank S Kellock and the Cassini team at Imperial College for MAG data processing, and GR Lewis, N Shane, and LK Gilbert at MSSL for ELS data processing. The French co-authors acknowledge support from CNRS and CNES.

[30] Masaki Fujimoto thanks W. R. Pryor and another reviewer for their assistance in evaluating this paper.

\section{References}

Badman, S. V., S. W. H. Cowley, J.-C. Gérard, and D. Grodent (2006), A statistical analysis of the location and width of Saturn's southern auroras, Ann. Geophys., 24, 3533-3545, doi:10.5194/angeo-24-3533-2006.

Badman, S. V., S. W. H. Cowley, L. Lamy, B. Cecconi, and P. Zarka (2008), Relationship between solar wind corotating interaction regions and the phasing and intensity of Saturn kilometric radiation bursts, Ann. Geophys., 26, 3641-3651, doi:10.5194/angeo-26-3641-2008.

Boudouridis, A., E. Zesta, L. R. Lyons, P. C. Anderson, and D. Lummerzheim (2004), Magnetospheric reconnection driven by solar wind pressure fronts, Ann. Geophys., 22, 1367-1378, doi:10.5194/angeo-22-1367-2004.

Bunce, E. J., S. W. H. Cowley, I. I. Alexeev, C. S. Arridge, M. K. Dougherty, J. D. Nichols, and C. T. Russell (2007), Cassini observations of the variation of Saturn's ring current parameters with system size, J. Geophys. Res., 112, A10202, doi:10.1029/2007JA012275.

Bunce, E. J., et al. (2008), Origin of Saturn's aurora: Simultaneous observations by Cassini and the Hubble Space Telescope, J. Geophys. Res., 113, A09209, doi:10.1029/2008JA013257.

Clarke, J. T., et al. (2005), Morphological differences between Saturn's ultraviolet aurorae and those of Earth and Jupiter, Nature, 433, 717-719, doi:10.1038/nature03331.

Clarke, J. T., et al. (2009), The response of Jupiter's and Saturn's auroral activity to the solar wind, J. Geophys. Res., 114, A05210, doi:10.1029 2008JA013694.

Cowley, S. W. H., S. V. Badman, E. J. Bunce, J. T. Clarke, J.-C. Gérard, D. Grodent, C. M. Jackman, S. E. Milan, and T. K. Yeoman (2005), Reconnection in a rotation-dominated magnetosphere and its relation to Saturn's auroral dynamics, J. Geophys. Res., 110, A02201, doi:10.1029/ 2004JA010796.

Dougherty, M. K., et al. (2004), The Cassini magnetic field investigation, Space Sci. Rev., 114, 331-383, doi:10.1007/s11214-004-1432-2.

Dougherty, M. K., et al. (2005), Cassini magnetometer observations during Saturn orbit insertion, Science, 307, 1266-1270, doi:10.1126/ science. 1106098 .

Grodent, D., J.-C. Gérard, S. W. H. Cowley, E. J. Bunce, and J. T. Clarke (2005), Variable morphology of Saturn's southern ultraviolet aurora, J. Geophys. Res., 110, A07215, doi:10.1029/2004JA010983.

Gurnett, D. A., et al. (2004), The Cassini Radio and plasma wave investigation, Space Sci. Rev., 114, 395-463, doi:10.1007/s11214-004-1434-0.

Jackman, C. M., N. Achilleos, E. J. Bunce, B. Cecconi, J. T. Clarke, S. W. H. Cowley, W. S. Kurth, and P. Zarka (2005), Interplanetary conditions and magnetospheric dynamics during the Cassini orbit insertion fly through of Saturn's magnetosphere, J. Geophys. Res., 110, A10212, doi:10.1029/ 2005JA011054.

Jackman, C. M., L. Lamy, M. P. Freeman, P. Zarka, B. Cecconi, W. S. Kurth, S. W. H. Cowley, and M. K. Dougherty (2009), On the character and distribution of lower-frequency radio emissions at Saturn and their relationship to substorm-like events, J. Geophys. Res., 114, A08211, doi:10.1029/2008JA013997.

Knight, S. (1973), Parallel electric fields, Planet. Space Sci., 21, 741-750, doi:10.1016/0032-0633(73)90093-7.

Kopf, A. J., et al. (2010), Electron beams as the source of auroral hiss at Saturn, Geophys. Res. Lett., 37, L09102, doi:10.1029/2010GL042980.

Kurth, W. S., et al. (2005), An Earth-like correspondence between Saturn's auroral features and radio emission, Nature, 433, 722-725, doi:10.1038/ nature03334.

Lamy, L., P. Zarka, B. Cecconi, R. Prange, W. S. Kurth, and D. A. Gurnett (2008), Saturn kilometric radiation: Average and statistical properties, J. Geophys. Res., 113, A07201, doi:10.1029/2007JA012900.

Lamy, L., B. Cecconi, R. Prangé, P. Zarka, J. D. Nichols, and J. T. Clarke (2009), An auroral oval at the footprint of Saturn's kilometric radio sources, co-located with the UV aurorae, J. Geophys. Res., 114, A10212, doi:10.1029/2009JA014401.

Lamy, L., et al. (2010), SKR properties measured within its source region: Local plasma, radio source location and diagram of emission, Geophys. Res. Lett., 37, L12104, doi:10.1029/2010GL043415.

Schippers, P., et al. (2008), Multi-instrument analysis of electron populations in Saturn's magnetosphere, J. Geophys. Res., 113, A07208, doi:10.1029/2008JA013098.

Talboys, D. L., C. S. Arridge, E. J. Bunce, A. J. Coates, S. W. H. Cowley, and M. K. Dougherty (2009a), Characterization of auroral current systems in Saturn's magnetosphere: High-latitude Cassini observations, J. Geophys. Res., 114, A06220, doi:10.1029/2008JA013846.

Talboys, D. L., C. S. Arridge, E. J. Bunce, A. J. Coates, S. W. H. Cowley, M. K. Dougherty, and K. K. Khurana (2009b), Signatures of fieldaligned currents in Saturn's nightside magnetosphere, Geophys. Res. Lett., 36, L19107, doi:10.1029/2009GL039867.

Ye, S.-Y., J. D. Menietti, G. Fischer, Z. Wang, B. Cecconi, D. A. Gurnett, and W. S. Kurth (2010), Z-mode waves as the source of Saturn narrowband radio emissions, J. Geophys. Res., 115, A08228, doi:10.1029/ 2009JA015167.

Young, D. T., et al. (2005), Composition and dynamics of plasma in Saturn's magnetosphere, Science, 307, 1262-1266, doi:10.1126/science.1106151.

C. S. Arridge and A. J. Coates, Mullard Space Science Laboratory, University College London, Dorking, RH5 6NT, UK.

E. J. Bunce, S. W. H. Cowley, and D. L. Talboys, Department of Physics and Astronomy, University of Leicester, Leicester, LE1 7RH, UK. (emma. bunce@ion.le.ac.uk)

B. Cecconi and P. Zarka, LESIA, CNRS, Observatoire de Paris, 5 Place Jules Janssen, 92190 Meudon, France.

M. K. Dougherty and L. Lamy, Blackett Laboratory, Imperial College, London SW7 2BZ, UK

W. S. Kurth and P. Schippers, Department of Physics and Astronomy, University of Iowa, Iowa City, IA 52242, USA. 